\title{
GEOMETRIC REALIZATIONS OF CURVATURE
}

\author{
M. BROZOS-VÁZQUEZ, P. GILKEY, AND S. NIKČEVIĆ
}

\begin{abstract}
We study geometric realization questions of curvature in the affine, Riemannian, almost Hermitian, almost para Hermitian, almost hyper Hermitian, almost hyper para Hermitian, Hermitian, and para Hermitian settings. We also express questions in Ivanov-Petrova geometry, Osserman geometry, and curvature homogeneity in terms of geometric realizations. $M S C: 53 \mathrm{~B} 20$
\end{abstract}

This paper is dedicated to Professor Sekigawa

\section{INTRODUCTION}

A central area of study in Differential Geometry is the examination of the relationship between purely algebraic properties of the Riemann curvature tensor and the underlying geometric properties of the manifold. Many authors have worked in this area in recent years. Nevertheless, many fundamental questions remain unanswered.

It is frequently convenient to work first purely algebraically and pass later to the geometric setting; many questions in differential geometry can be phrased as problems involving the geometric realization of curvature. Here is a brief outline to this paper. In Section 2, we study the affine setting, in Section 3, we study pseudo Riemannian geometry, and in Section 4, we combine these two structures and consider realization problems related to affine geometry where the additional structure of a pseudo Riemannian metric is present. In Section 5, we turn our attention to almost Hermitian and almost para Hermitian geometry and study the scalar curvature and the $\star$-scalar curvature. In Section 6, we examine similar questions in hyper almost Hermitian geometry and hyper almost para Hermitian geometry. In Section 7. we study realization questions which arise when the structures in question are to be integrable. In Sections 8 and 9, we discuss Ivanov-Petrova geometry and Osserman geometry, respectively. In Section 10, we present questions of curvature homogeneity.

The decomposition of the appropriate space of tensors into irreducible modules under the appropriate structure group is central to our investigation and we review the appropriate results in each section. The results in Sections 2,7 although they involve non-linear analysis, are closely tied to the representation theory of the appropriate group. In contrast, the results of Sections 8, 10 are non-linear in their very formulation since one is studying orbit spaces under the structure group which are not linear subspaces. Throughout this paper, we shall let $M$ be a smooth manifold of dimension $m \geq 4$; there are similar results in the 2 dimensional and 3 dimensional settings. We shall let $\nabla$ be a torsion free connection on the tangent bundle of $M$. Let $g$ be a pseudo Riemannian metric of signature $(p, q)$ on $M$ and let $\mathcal{M}:=(M, g)$ be the associated pseudo Riemannian manifold.

\section{Affine Geometry}

We refer to 38, 66 for further information concerning affine geometry. An affine manifold is a pair $(M, \nabla)$ where $M$ is a smooth manifold and where $\nabla$ is a torsion 
free connection on $M$. The associated curvature operator $\mathcal{R}$ is defined by setting:

$$
\mathcal{R}(x, y):=\nabla_{x} \nabla_{y}-\nabla_{y} \nabla_{x}-\nabla_{[x, y]} .
$$

This $(1,3)$ tensor satisfies the identities:

$$
\mathcal{R}(x, y)=-\mathcal{R}(y, x), \quad \mathcal{R}(x, y) z+\mathcal{R}(y, z) x+\mathcal{R}(z, x) y=0 .
$$

Let $V$ be a vector space of dimension $m$. A tensor $\mathcal{A} \in \otimes^{2} V^{*} \otimes \operatorname{End}(V)$ satisfying the symmetries given in Equation (2.a) is called an affine algebraic curvature operator; let $\mathfrak{A}(V) \subset \otimes^{2} V^{*} \otimes \operatorname{End}(V)$ be the subspace of all such operators. An affine curvature operator $\mathcal{A} \in \mathfrak{A}(V)$ is said to be geometrically realizable if there exists an affine manifold $(M, \nabla)$, if there exists a point $P$ of $M$ (which is called the realizing point), and if there exists an isomorphism $\phi: V \rightarrow T_{P} M$ so that $\phi^{*} \mathcal{R}_{P}=\mathcal{A}$. In either the algebraic or the geometric setting, one defines the Ricci tensor $\rho$ by:

$$
\rho(x, y):=\operatorname{Tr}\{z \rightarrow \mathcal{A}(z, x) y\} .
$$

2.1. The decomposition of $\mathfrak{A}(V)$ as a $\mathrm{GL}(V)$ module. The action of the general linear group $\mathrm{GL}(V)$ on the vector space of affine algebraic curvature operators $\mathfrak{A}(V)$ by pullback is not irreducible, but decomposes as the direct sum of irreducible modules. The decomposition $V^{*} \otimes V^{*}=\Lambda^{2}\left(V^{*}\right) \oplus S^{2}\left(V^{*}\right)$ is a $\operatorname{GL}(V)$ equivariant decomposition of $V^{*} \otimes V^{*}$ into irreducible GL( $\left.V\right)$ modules; we let $\rho_{a}$ and $\rho_{s}$ be the components in $\Lambda^{2}\left(V^{*}\right)$ and $S^{2}\left(V^{*}\right)$, respectively, where

$$
\rho_{a}(x, y):=\frac{1}{2}\{\rho(x, y)-\rho(y, x)\} \quad \text { and } \quad \rho_{s}(x, y):=\frac{1}{2}\{\rho(x, y)+\rho(y, x)\} .
$$

One has the following result of Bokan [6] and of Strichartz [70]:

Theorem 2.1. Let $\operatorname{dim}(V) \geq 4$. The Ricci tensor $\rho$ yields a $\mathrm{GL}(V)$ equivariant short exact sequence

$$
0 \rightarrow \operatorname{ker}(\rho) \rightarrow \mathfrak{A}(V) \stackrel{\rho_{a} \oplus \rho_{s}}{\longrightarrow} \Lambda^{2}\left(V^{*}\right) \oplus S^{2}\left(V^{*}\right) \rightarrow 0
$$

which is equivariantly split by the map $\sigma$ where

$$
\begin{aligned}
& \left\{\sigma \rho_{a}\right\}(x, y) z=\frac{-1}{1+m}\left\{2 \rho_{a}(x, y) z+\rho_{a}(x, z) y-\rho_{a}(y, z) x\right\}, \\
& \left\{\sigma \rho_{s}\right\}(x, y) z=\frac{1}{1-m}\left\{\rho_{s}(x, z) y-\rho_{s}(y, z) x\right\} .
\end{aligned}
$$

One has a direct sum decomposition of $\mathfrak{A}(V)$ into irreducible $\mathrm{GL}(V)$ modules:

$$
\mathfrak{A}(V)=\operatorname{ker}(\rho) \oplus \Lambda^{2}\left(V^{*}\right) \oplus S^{2}\left(V^{*}\right) .
$$

We note for the sake of completeness that:

$$
\begin{array}{|l|l|}
\hline \operatorname{dim}\{\operatorname{ker}(\rho) \cap \mathfrak{A}(V)\}=\frac{m^{2}\left(m^{2}-4\right)}{3} & \operatorname{dim}\left\{\Lambda^{2}\left(V^{*}\right)\right\}=\frac{m(m-1)}{2} \\
\hline \operatorname{dim}\left\{S^{2}\left(V^{*}\right)\right\}=\frac{m(m+1)}{2} & \operatorname{dim}\{\mathfrak{A}(V)\}=\frac{m^{2}\left(m^{2}-1\right)}{3} \\
\hline
\end{array}
$$

Definition 2.2. Let $\mathcal{A} \in \mathfrak{A}(V)$.

(1) $\mathcal{A}$ is Ricci symmetric if and only if $\rho \in S^{2}\left(V^{*}\right)$, i.e. $\rho_{a}=0$.

(2) $\mathcal{A}$ is Ricci anti-symmetric if and only if $\rho \in \Lambda^{2}\left(V^{*}\right)$, i.e. $\rho_{s}=0$.

(3) $\mathcal{A}$ is Ricci flat if and only if $\rho=0$.

(4) The Weyl projective curvature operator $\mathcal{P}$ is the component of $\mathcal{A}$ in $\operatorname{ker}(\rho)$, i.e. $\mathcal{P}:=\mathcal{A}-\sigma \rho \in \operatorname{ker} \rho$. $\mathcal{A}$ is projectively flat if and only if $\mathcal{P}=0$.

(5) $\mathcal{A}$ is flat if and only if $\mathcal{A}=0$, i.e. $\mathcal{A}$ is both projectively flat and Ricci flat. 
2.2. Equiaffine geometry. Ricci symmetric torsion free connections are often called equiaffine; they play a central role in many settings - see, for example, the discussion in [5, 7, 46, 50, 57. The following result is well known 61. and motivates their investigation:

Theorem 2.3. Let $(M, \nabla)$ be an affine manifold. The following assertions are equivalent:
(1) $\operatorname{Tr}(\mathcal{R})=0$
(2) $\nabla$ is Ricci symmetric.
(3) $\nabla$ locally admits a parallel volume form.

2.3. Geometric realizability of affine algebraic curvature operators I. Theorem 2.1 gives rise to additional geometric realizability questions; the decomposition of $\mathfrak{A}(V)$ as a $\mathrm{GL}(V)$ module has 3 components so there are 8 natural geometric realization questions which are $\operatorname{GL}(V)$ equivariant. We refer to the discussion in [37. 39] for the proof of the following result which shows, in particular, that the symmetries of Equation (2.a) generate the universal symmetries of the curvature operator of a torsion free connection:

\section{Theorem 2.4.}

(1) Any affine algebraic curvature operator can be geometrically realized by an affine manifold.

(2) Any Ricci symmetric affine algebraic curvature operator can be geometrically realized by a Ricci symmetric affine manifold.

(3) Any Ricci anti-symmetric affine algebraic curvature operator can be geometrically realized by a Ricci anti-symmetric affine manifold.

(4) Any Ricci flat affine algebraic curvature operator can be geometrically realized by a Ricci flat affine manifold.

(5) Any projectively flat affine algebraic curvature operator can be geometrically realized by a projectively flat affine manifold.

(6) Any projectively flat Ricci symmetric affine algebraic curvature operator can be geometrically realized by a projectively flat Ricci symmetric affine manifold.

(7) A projectively flat Ricci anti-symmetric affine algebraic curvature operator which is not flat can not be geometrically realized by a projectively flat, Ricci anti-symmetric affine manifold.

(8) If $\mathcal{A}$ is flat, then $\mathcal{A}$ is geometrically realized by a flat affine manifold.

These geometric realizability results can be summarized in the following table; the non-zero components of $\mathcal{A}$ are indicated by $\star$.

\begin{tabular}{|c|c|c|c|c|c|c|c|}
\hline $\operatorname{ker}(\rho)$ & $S^{2}\left(V^{*}\right)$ & $\Lambda^{2}\left(V^{*}\right)$ & & $\operatorname{ker}(\rho)$ & $S^{2}\left(V^{*}\right)$ & $\Lambda^{2}\left(V^{*}\right)$ & \\
\hline$\star$ & $\star$ & $\star$ & yes & 0 & $\star$ & $\star$ & yes \\
\hline$\star$ & $\star$ & 0 & yes & 0 & $\star$ & 0 & yes \\
\hline$\star$ & 0 & $\star$ & yes & 0 & 0 & $\star$ & no \\
\hline$\star$ & 0 & 0 & yes & 0 & 0 & 0 & yes \\
\hline
\end{tabular}

\section{Pseudo Riemannian Geometry}

Let $V$ be a finite dimensional real vector space of dimension $m$. One says that $A \in \otimes^{4}\left(V^{*}\right)$ is an algebraic curvature tensor on $V$ if $A$ satisfies the symmetries of the Riemann curvature tensor:

$$
\begin{aligned}
& A(x, y, z, w)=-A(y, x, z, w)=A(z, w, x, y), \\
& A(x, y, z, w)+A(y, z, x, w)+A(z, x, y, w)=0 .
\end{aligned}
$$


Let $\mathfrak{R}(V)$ be the space of all such 4 -tensors; note that $\langle\cdot, \cdot\rangle$ induces a non-degenerate innerproduct on $\mathfrak{R}(V)$. We say that $\mathfrak{M}:=(V,\langle\cdot, \cdot\rangle, A)$ is a curvature model if $A \in \mathfrak{R}(V)$ and if $\langle\cdot, \cdot\rangle$ is a non-degenerate symmetric bilinear form of signature $(p, q)$ on $V$. $\mathfrak{M}$ is said to be Riemannian if $p=0$ and Lorentzian if $p=1$. Two curvature models $\mathfrak{M}_{1}=\left(V_{1},\langle\cdot, \cdot\rangle_{1}, A_{1}\right)$ and $\mathfrak{M}_{2}=\left(V_{2},\langle\cdot, \cdot\rangle_{2}, A_{2}\right)$ are said to be isomorphic, and one writes $\mathfrak{M}_{1} \approx \mathfrak{M}_{2}$, if there is an isomorphism $\phi: V_{1} \rightarrow V_{2}$ so that

$$
\phi^{*}\langle\cdot, \cdot\rangle_{2}=\langle\cdot, \cdot\rangle_{1} \quad \text { and } \quad \phi^{*} A_{2}=A_{1} .
$$

3.1. The decomposition of $\mathfrak{R}(V)$ as an $O(V,\langle\cdot, \cdot\rangle)$ module. If $\mathfrak{M}$ is a curvature model, then the associated orthogonal group $O(V,\langle\cdot, \cdot\rangle)$ is defined by setting:

$$
O(V,\langle\cdot, \cdot\rangle):=\left\{T \in \mathrm{GL}(V): T^{*}\langle\cdot, \cdot\rangle=\langle\cdot, \cdot\rangle\right\} .
$$

Let $\varepsilon_{i j}$ and $A_{i j k l}$ be the components of $\langle\cdot, \cdot\rangle$ and of $A$ relative to a basis $\left\{e_{i}\right\}$ for $V$ :

$$
\varepsilon_{i j}:=\left\langle e_{i}, e_{j}\right\rangle \text { and } A_{i j k l}:=A\left(e_{i}, e_{j}, e_{k}, e_{l}\right) \text {. }
$$

Let $\varepsilon^{i j}$ be the inverse matrix. Adopt the Einstein convention and sum over repeated indices. The components of the Ricci tensor $\rho$ and the scalar curvature $\tau$ are:

$$
\rho_{i l}:=\varepsilon^{j k} A_{i j k l} \quad \text { and } \quad \tau:=\varepsilon^{i l} \rho_{i l} .
$$

Let $S_{0}^{2}\left(V^{*},\langle\cdot, \cdot\rangle\right) \subset S^{2}\left(V^{*}\right)$ be the subspace of trace free symmetric 2-tensors and let $\rho_{0}:=\rho-\frac{1}{m} \tau\langle\cdot, \cdot\rangle$ be the trace free Ricci tensor. We refer to Singer and Thorpe 69 for:

Theorem 3.1. Let $\operatorname{dim}(V) \geq 4$. There is an $O(V,\langle\cdot, \cdot\rangle)$ equivariant short exact sequence

$$
0 \rightarrow \operatorname{ker}(\rho) \rightarrow \mathfrak{R}(V) \stackrel{\rho_{0} \oplus \tau}{\longrightarrow} S_{0}^{2}\left(V^{*},\langle\cdot, \cdot\rangle\right) \oplus \mathbb{R} \rightarrow 0
$$

which is equivariantly split by the map $\sigma$ where

$$
\begin{aligned}
\sigma(\rho)(x, y, z, w): & =\frac{1}{m-2}\{\rho(x, w)\langle y, z\rangle+\langle x, w\rangle \rho(y, z)\} \\
& -\frac{1}{m-2}\{\rho(x, z)\langle y, w\rangle+\langle x, z\rangle \rho(y, w)\} \\
& -\frac{\tau}{(m-1)(m-2)}\{\langle x, w\rangle\langle y, z\rangle-\langle x, z\rangle\langle y, w\rangle\} .
\end{aligned}
$$

One has an orthogonal decomposition of $\mathfrak{R}(V)$ into irreducible $O(V,\langle\cdot, \cdot\rangle)$ modules:

$$
\mathfrak{R}(V)=\operatorname{ker}(\rho) \oplus S_{0}^{2}\left(V^{*},\langle\cdot, \cdot\rangle\right) \oplus \mathbb{R} .
$$

We note for the sake of completeness that:

\begin{tabular}{|l|l|}
\hline $\operatorname{dim}\{\mathbb{R}\}=1$ & $\operatorname{dim}\{\operatorname{ker}(\rho) \cap \mathfrak{R}(V)\}=\frac{m(m+1)(m+2)(m-3)}{12}$ \\
\hline $\operatorname{dim}\{\mathfrak{R}(V)\}=\frac{m^{2}\left(m^{2}-1\right)}{12}$ & $\operatorname{dim}\left\{S_{0}^{2}\left(V^{*},\langle\cdot, \cdot\rangle\right)\right\}=\frac{(m-1)(m+2)}{2}$ \\
\hline
\end{tabular}

3.2. Geometric realizability of algebraic curvature tensors. Assume given a pseudo Riemannian manifold $\mathcal{M}:=(M, g)$ of signature $(p, q)$. Let $\nabla$ be the Levi-Civita connection of $\mathcal{M}$ and let $R \in \otimes^{4} T^{*} M$ be the curvature tensor:

$$
R(x, y, z, w)=g(\mathcal{R}(x, y) z, w) .
$$

Let $\mathfrak{M}=(V,\langle\cdot, \cdot\rangle, A)$ be a curvature model. We say that $\mathfrak{M}$ is geometrically realizable if thereexists a pseudo Riemannian manifold $\mathcal{M}$, if there exists a point $P$ of $M$, and if there exists an isomorphism $\phi: V \rightarrow T_{P} M$ so that

$$
\phi^{*} g_{P}=\langle\cdot, \cdot\rangle \text { and } \phi^{*} R_{P}=A \text {. }
$$

The Weyl conformal curvature tensor $W:=A-\sigma \rho$ is the projection of $A$ on $\operatorname{ker}(\rho)$; we say a model or a pseudo Riemannian manifold is conformally flat if and only if $W=0$. The following result 11 shows, in particular, that the relations of Equation (3.a) generate the universal symmetries of the Riemann curvature tensor. We focus our attention on the scalar curvature: 


\section{Theorem 3.2.}

(1) Any curvature model is geometrically realizable by a pseudo Riemannian manifold of constant scalar curvature.

(2) Any conformally flat curvature model is geometrically realizable by a conformally flat pseudo Riemannian manifold of constant scalar curvature.

To solve the Yamabe problem [3, 62, 74, 76], one constructs a Riemannian metric of constant scalar curvature in a given conformal class on a compact Riemannian manifold. The complex analogue also has been solved [21] by constructing an almost Hermitian metric of constant scalar curvature in the conformal class of a compact almost Hermitian manifold. Theorem 3.2 has a somewhat different flavor as we are not fixing the conformal class but rather the curvature tensor at the realizing point. Furthermore, our manifolds are not compact nor even complete.

\section{Affine and Riemannian Geometry}

We now consider mixed structures - we shall study an affine structure and a pseudo-Riemannian metric where the given affine connection is not the Levi-Civita connection of the pseudo-Riemannian metric; thus the two structures are decoupled.

Let $\langle\cdot, \cdot\rangle$ be a non-degenerate symmetric inner product on $V$ of signature $(p, q)$. Expand $\mathcal{A} \in \mathfrak{A}(V)$ in the form

$$
\mathcal{A}\left(e_{i}, e_{j}\right) e_{k}=\mathcal{A}_{i j k}{ }^{\ell} e_{\ell} .
$$

The scalar curvature $\tau$ and trace free Ricci tensor $\rho_{0}$ are then given, respectively, by contracting indices

$$
\tau:=\varepsilon^{i j} \mathcal{A}_{k i j}{ }^{k}, \quad \rho_{0}(x, y):=\rho_{s}(x, y)-\frac{\tau}{m}\langle x, y\rangle .
$$

One has an orthogonal decomposition of $V^{*} \otimes V^{*}$ into irreducible $O(V,\langle\cdot, \cdot\rangle)$ modules

$$
V^{*} \otimes V^{*}=\Lambda^{2}\left(V^{*}\right) \oplus S_{0}^{2}\left(V^{*},\langle\cdot, \cdot\rangle\right) \oplus \mathbb{R} .
$$

4.1. Geometric realizability of affine algebraic curvature tensors II. The decomposition of Equation (4.a) leads to several geometric realization questions which are natural with respect to the structure group $O(V,\langle\cdot, \cdot\rangle)$ and which can all be solved either in the real analytic category or in the $C^{s}$ category for any $s \geq 1$. As our considerations are local, we take $M=V$ and $P=0$. The primary focus of our investigation is on constant scalar curvature and on properties of the Ricci tensor. We refer to [40] for the proof of the following result:

Theorem 4.1. Let $g$ be a $C^{s}$ (resp. real analytic) pseudo Riemannian metric which is defined on an open neighborhood of $0 \in V$. Let $\mathcal{A} \in \mathfrak{A}(V)$. There exists a torsion free $C^{s}$ (resp. real analytic) connection $\nabla$ which is defined on a smaller open neighborhood of 0 in $V$ such that:

(1) $\mathcal{R}_{0}=\mathcal{A}$.

(2) $\nabla$ has constant scalar curvature.

(3) If $\mathcal{A}$ is Ricci symmetric, then $\nabla$ is Ricci symmetric.

(4) If $\mathcal{A}$ is Ricci anti-symmetric, then $\nabla$ is Ricci anti-symmetric.

(5) If $\mathcal{A}$ is Ricci traceless, then $\nabla$ is Ricci traceless.

4.2. The decomposition of $\mathfrak{A}(V)$ as an $O(V,\langle\cdot, \cdot\rangle)$ module. The subspace $\operatorname{ker}(\rho) \subset \mathfrak{A}(V)$ is not an irreducible $O(V,\langle\cdot, \cdot\rangle)$ module but decomposes as the direct sum of 5 additional irreducible factors. We refer to Bokan $[6]$ for the proof of the following result:

Theorem 4.2. Let $\operatorname{dim}(V) \geq 4$. There is an orthogonal decomposition of $\mathfrak{A}(V)$ into 8 irreducible $O(V,\langle\cdot, \cdot\rangle)$ modules $\mathfrak{A}(V)=A_{1} \oplus \ldots \oplus A_{8}$ where: 


$$
\begin{aligned}
& A_{1} \approx \mathbb{R}, \quad A_{2} \approx A_{5} \approx S_{0}^{2}\left(V^{*},\langle\cdot, \cdot\rangle\right), \quad A_{3} \approx A_{4} \approx \Lambda^{2}\left(V^{*}\right), \\
& A_{6}=\left\{\Theta \in \otimes^{4} V^{*}: \Theta_{i j k l}+\Theta_{j k i l}+\Theta_{k i j l}=0, \Theta_{i j k l}=-\Theta_{j i k l}=\Theta_{k l i j}\right. \text {, } \\
& \left.\varepsilon^{i l} \Theta_{i j k l}=0\right\}, \\
& A_{7}=\left\{\Theta \in \otimes^{4} V^{*}: \Theta_{k j i l}+\Theta_{i k j l}-\Theta_{l j i k}-\Theta_{i l j k}=0, \Theta_{i j k l}=-\Theta_{j i k l}=\Theta_{i j l k},\right. \\
& \left.\varepsilon^{i l} \Theta_{i j k l}=0\right\}, \\
& A_{8}=\left\{\Theta \in \otimes^{4} V^{*}: \Theta_{i j k l}=-\Theta_{j i k l}=-\Theta_{i j l k}=-\Theta_{k l i j}, \varepsilon^{i l} \Theta_{i j k l}=0\right\} .
\end{aligned}
$$

We note for the sake of completeness that:

\begin{tabular}{|l|l|}
\hline $\operatorname{dim}\left\{W_{2}\right\}=\operatorname{dim}\left\{W_{5}\right\}=\frac{(m-1)(m+2)}{2}$ & $\operatorname{dim}\left\{W_{1}\right\}=1$ \\
\hline $\operatorname{dim}\left\{W_{3}\right\}=\operatorname{dim}\left\{W_{4}\right\}=\frac{m(m-1)}{2}$ & $\operatorname{dim}\left\{W_{6}\right\}=\frac{m(m+1)(m-3)(m+2)}{12}$ \\
\hline $\operatorname{dim}\left\{W_{7}\right\}=\frac{(m-1)(m-2)(m+1)(m+4)}{8}$ & $\operatorname{dim}\left\{W_{8}\right\}=\frac{m(m-1)(m-3)(m+2)}{8}$ \\
\hline
\end{tabular}

\section{5. almost Hermitian Geometry}

We refer to the discussion in 22, 24, 25, 47, 49, 60, 64 for additional information concerning almost Hermitian geometry. We refer to 20 for further information concerning almost para Hermitian geometry; for example, para Hermitian geometry enters in the study of Osserman Walker metrics of signature $(2,2)$ 23], it is important in the study of homogeneous geometries [28, and it is relevant to the study of Walker manifolds with degenerate self-dual Weyl curvature operators [18.

Let $J$ be a linear map of $V$ and let $\mathfrak{M}=(V,\langle\cdot, \cdot\rangle, A)$ be a curvature model. One says that $J$ is a Hermitian structure if

$$
J^{2}=- \text { id } \quad \text { and } \quad J^{*}\langle\cdot, \cdot\rangle=\langle\cdot, \cdot\rangle .
$$

Similarly, one says that $J$ is a para Hermitian structure if

$$
J^{2}=\text { id } \text { and } J^{*}\langle\cdot, \cdot\rangle=-\langle\cdot, \cdot\rangle .
$$

Note that Hermitian structures exist if and only if both $p$ and $q$ are even; para Hermitian structures exist if and only if $p=q$. Let $\mathfrak{C}:=(V,\langle\cdot, \cdot\rangle, J, A)$ be the associated Hermitian curvature model (resp. para Hermitian curvature model). Define the $\star$-Ricci tensor $\rho^{\star}$ and the $\star$-scalar curvature $\tau^{\star}$ in either case, by setting:

$$
\begin{aligned}
& \rho^{\star}(x, y):=\left\{\begin{array}{ccl}
\varepsilon^{i l} A\left(e_{i}, x, J y, J e_{l}\right) & \text { if } & \mathfrak{C} \text { is Hermitian, } \\
-\varepsilon^{i l} A\left(e_{i}, x, J y, J e_{l}\right) & \text { if } & \mathfrak{C} \text { is para Hermitian, }
\end{array}\right. \\
& \tau^{\star}:=\left\{\begin{array}{ccl}
\varepsilon^{i l} \varepsilon^{j k} A\left(e_{i}, e_{j}, J e_{k}, J e_{l}\right) & \text { if } & \mathfrak{C} \text { is Hermitian, } \\
-\varepsilon^{i l} \varepsilon^{j k} A\left(e_{i}, e_{j}, J e_{k}, J e_{l}\right) & \text { if } & \mathfrak{C} \text { is para Hermitian. }
\end{array}\right.
\end{aligned}
$$

5.1. The geometric realizability of almost Hermitian models. One says that a manifold $\mathcal{C}:=(M, g, J)$ is an almost Hermitian manifold (resp. almost para Hermitian manifold) if $\left(T_{P} M, g_{P}, J_{P}, R_{P}\right)$ is a Hermitian (resp. para Hermitian) curvature model for every $P \in M$. We do not assume that the structure $J$ on $M$ is integrable as this imposes additional curvature identities [41] as we shall see presently. The notion of geometricrealizability in this context is defined similarly. Again, we focus our attention on the scalar curvature and the analogous $\star$-scalar curvature in the following Theorem [1]:

Theorem 5.1. Let $m \geq 4$.

(1) Any Hermitian curvature model is geometrically realizable by an almost Hermitian manifold of constant scalar and constant $\star$-scalar curvature.

(2) Any para Hermitian curvature model is geometrically realizable by an almost para Hermitian manifold of constant scalar and constant $\star$-scalar curvature. 
5.2. The decomposition of $\mathfrak{R}(V)$ as a unitary module. Let $(V,\langle\cdot, \cdot\rangle, J)$ be a Hermitian structure. The Kaehler form is defined by $\Omega(x, y):=\langle x, J y\rangle$. Set

$$
\begin{aligned}
& S_{0,+}=S_{0,+}^{2}\left(V^{*},\langle\cdot, \cdot\rangle, J\right):=\left\{\theta \in S^{2}\left(V^{*}\right): J^{*} \theta=\theta, \theta \perp\langle\cdot, \cdot\rangle\right\}, \\
& \Lambda_{0,+}=\Lambda_{0,+}^{2}\left(V^{*},\langle\cdot, \cdot\rangle, J\right):=\left\{\theta \in \Lambda^{2}\left(V^{*}\right): J^{*} \theta=\theta, \theta \perp \Omega\right\}, \\
& S_{-}^{2}=S_{-}^{2}\left(V^{*}, J\right):=\left\{\theta \in S^{2}\left(V^{*}\right): J^{*} \theta=-\theta\right\}, \\
& \Lambda_{-}^{2}=\Lambda_{-}^{2}\left(V^{*}, J\right):=\left\{\theta \in \Lambda^{2}\left(V^{*}\right): J^{*} \theta=-\theta\right\} .
\end{aligned}
$$

Let $\mathcal{U}(V,\langle\cdot, \cdot\rangle, J)$ be the associated unitary group:

$$
\mathcal{U}(V,\langle\cdot, \cdot\rangle, J):=\left\{U \in \mathrm{GL}(V): U J=J U \quad \text { and } \quad U^{*}\langle\cdot, \cdot\rangle=\langle\cdot, \cdot\rangle\right\} .
$$

We have the following decomposition of $V^{*} \otimes V^{*}$ as the orthogonal direct sum of irreducible $\mathcal{U}(V,\langle\cdot, \cdot\rangle, J)$ modules:

$$
V \otimes V^{*}=\langle\cdot, \cdot\rangle \cdot \mathbb{R} \oplus S_{0,+}^{2} \oplus S_{-}^{2} \oplus \Omega \cdot \mathbb{R} \oplus \Lambda_{0,+}^{2} \oplus \Lambda_{-}^{2} .
$$

We let $\rho_{0,+, S}, \rho_{0,+, S}^{\star}, \rho_{-, S}$, and $\rho_{-, \Lambda}^{\star}$ denote the components of $\rho$ and $\rho^{\star}$ with respect to this decomposition.

We refer to 72 for the proof of Theorem 5.2 in the Riemannian setting - the extension to the higher signature context is not difficult [12. This result has been used by many authors [15, 27, 29, 30, 151.

Theorem 5.2. Let $(V,\langle\cdot, \cdot\rangle, J)$ be a Hermitian structure.

(1) We have the following orthogonal direct sum decomposition of $\mathfrak{R}(V)$ into irreducible $\mathcal{U}(V,\langle\cdot, \cdot\rangle, J)$ modules:

(a) If $2 n=4, \mathfrak{R}(V)=\mathcal{W}_{1} \oplus \mathcal{W}_{2} \oplus \mathcal{W}_{3} \oplus \mathcal{W}_{4} \oplus \mathcal{W}_{7} \oplus \mathcal{W}_{8} \oplus \mathcal{W}_{9}$.

(b) If $2 n=6, \mathfrak{R}(V)=\mathcal{W}_{1} \oplus \mathcal{W}_{2} \oplus \mathcal{W}_{3} \oplus \mathcal{W}_{4} \oplus \mathcal{W}_{5} \oplus \mathcal{W}_{7} \oplus \mathcal{W}_{8} \oplus \mathcal{W}_{9} \oplus \mathcal{W}_{10}$.

(c) If $2 n \geq 8, \mathfrak{R}(V)=\mathcal{W}_{1} \oplus \mathcal{W}_{2} \oplus \mathcal{W}_{3} \oplus \mathcal{W}_{4} \oplus \mathcal{W}_{5} \oplus \mathcal{W}_{6} \oplus \mathcal{W}_{7} \oplus \mathcal{W}_{8} \oplus \mathcal{W}_{9} \oplus \mathcal{W}_{10}$.

We have $\mathcal{W}_{1} \approx \mathcal{W}_{4}$ and, if $2 n \geq 6, \mathcal{W}_{2} \approx \mathcal{W}_{5}$. The other $\mathcal{U}(V,\langle\cdot, \cdot\rangle, J)$ modules appear with multiplicity 1.

(2) We have that:
(a) $\tau \oplus \tau^{\star}: \mathcal{W}_{1} \oplus \mathcal{W}_{4} \stackrel{\approx}{\longrightarrow} \mathbb{R} \oplus \mathbb{R}$.
(b) If $2 n=4, \rho_{0,+, S}: \mathcal{W}_{2} \stackrel{\approx}{\longrightarrow} S_{0,+}^{2}$.
(c) If $2 n \geq 6, \rho_{0,+, S} \oplus \rho_{0,+, S}^{\star}: \mathcal{W}_{2} \oplus \mathcal{W}_{5} \stackrel{\approx}{\longrightarrow} S_{0,+}^{2} \oplus S_{0,+}^{2}$.
(d) $\mathcal{W}_{3}=\{A \in \mathfrak{R}(V): A(x, y, z, w)=A(J x, J y, z, w) \forall x, y, z, w\} \cap \operatorname{ker}(\rho)$.
(e) If $2 n \geq 8, \mathcal{W}_{6}=\operatorname{ker}\left(\rho \oplus \rho^{\star}\right) \cap\left\{A \in \mathfrak{R}(V): J^{*} A=A\right\} \cap \mathcal{W}_{3}^{\perp}$.
(f) $\mathcal{W}_{7}=\{A \in \mathfrak{R}(V): A(J x, y, z, w)=A(x, y, J z, w) \forall x, y, z, w\}$.
(g) $\rho_{-, S}: \mathcal{W}_{8} \stackrel{\approx}{\longrightarrow} S_{-}^{2}$ and $\rho_{-, \Lambda}^{\star}: \mathcal{W}_{9} \stackrel{\approx}{\longrightarrow} \Lambda_{-}^{2}$.
(h) If $2 n \geq 6, \mathcal{W}_{10}=\left\{A \in \mathfrak{R}(V): J^{*} A=-A\right\} \cap \operatorname{ker}\left(\rho \oplus \rho^{\star}\right)$.

Let $m=\operatorname{dim}(V)=2 n$. We note for the sake of completeness that:

\begin{tabular}{|l|l|l|l||l|l|l|l|}
\hline & $m=4$ & $m=6$ & $m \geq 8$ & & $m=4$ & $m=6$ & $m \geq 8$ \\
\hline $\mathcal{W}_{1}$ & 1 & 1 & 1 & $\mathcal{W}_{2}$ & 3 & 8 & $n^{2}-1$ \\
\hline $\mathcal{W}_{3}$ & 5 & 27 & $\frac{n^{2}(n-1)(n+3)}{4}$ & $\mathcal{W}_{4}$ & 1 & 1 & 1 \\
\hline $\mathcal{W}_{6}$ & 0 & 0 & $\frac{n^{2}(n+1)(n-3)}{4}$ & $\mathcal{W}_{5}$ & 0 & 8 & $n^{2}-1$ \\
\hline $\mathcal{W}_{7}$ & 2 & 12 & $\frac{n^{2}\left(n^{2}-1\right)}{6}$ & $\mathcal{W}_{8}$ & 6 & 12 & $n^{2}+n$ \\
\hline $\mathcal{W}_{10}$ & 0 & 30 & $\frac{2 n^{2}\left(n^{2}-4\right)}{3}$ & $\mathcal{W}_{9}$ & 2 & 6 & $n^{2}-n$ \\
\hline
\end{tabular}


5.3. The decomposition of $\mathfrak{R}(V)$ as a para unitary module. We change the signs appropriately to obtain a corresponding decomposition in the para Hermitian context. Let $(V,\langle\cdot, \cdot\rangle, \tilde{J})$ be a para Hermitian structure. Let $\tilde{\Omega}(x, y):=\langle x, \tilde{J} y\rangle$ be the para Kaehler form. We have

$$
\tilde{J}^{*} \tilde{\Omega}=-\tilde{\Omega} \quad \text { and } \quad \tilde{J}^{*}\langle\cdot, \cdot\rangle=-\langle\cdot, \cdot\rangle .
$$

Set

$$
\begin{aligned}
& S_{+}^{2}=S_{+}^{2}\left(V^{*}, \tilde{J}\right):=\left\{\theta \in S^{2}\left(V^{*}\right): \tilde{J}^{*} \theta=\theta\right\}, \\
& \Lambda_{+}^{2}=\Lambda_{+}^{2}\left(V^{*}, \tilde{J}\right):=\left\{\theta \in \Lambda^{2}\left(V^{*}\right): \tilde{J}^{*} \theta=\theta\right\}, \\
& S_{0,-}^{2}=S_{0,-}^{2}\left(V^{*},\langle\cdot, \cdot\rangle, \tilde{J}\right):=\left\{\theta \in S^{2}\left(V^{*}\right): \tilde{J}^{*} \theta=-\theta, \theta \perp\langle\cdot, \cdot\rangle\right\}, \\
& \Lambda_{0,-}^{2}=\Lambda_{0,-}^{2}\left(V^{*},\langle\cdot, \cdot\rangle, \tilde{J}\right):=\left\{\theta \in \Lambda^{2}\left(V^{*}\right): \tilde{J}^{*} \theta=-\theta, \theta \perp \tilde{\Omega}\right\}, \\
& \tilde{\mathcal{U}}(V,\langle\cdot, \cdot\rangle, J):=\left\{\tilde{U} \in \operatorname{GL}(V): \tilde{U} \tilde{J}=\tilde{J} \tilde{U} \quad \text { and } \quad \tilde{U}^{*}\langle\cdot, \cdot\rangle=\langle\cdot, \cdot\rangle\right\} .
\end{aligned}
$$

Theorem 5.3. Let $(V,\langle\cdot, \cdot\rangle, \tilde{J})$ be a para Hermitian structure.

(1) We have the following orthogonal direct sum decomposition of $\mathfrak{R}(V)$ into irreducible $\tilde{\mathcal{U}}(V,\langle\cdot, \cdot\rangle, J)$ modules:

(a) If $2 n=4, \mathfrak{R}(V)=\tilde{\mathcal{W}}_{1} \oplus \tilde{\mathcal{W}}_{2} \oplus \tilde{\mathcal{W}}_{3} \oplus \tilde{\mathcal{W}}_{4} \oplus \tilde{\mathcal{W}}_{7} \oplus \tilde{\mathcal{W}}_{8} \oplus \tilde{\mathcal{W}}_{9}$.

(b) If $2 n=6, \mathfrak{R}(V)=\tilde{\mathcal{W}}_{1} \oplus \tilde{\mathcal{W}}_{2} \oplus \tilde{\mathcal{W}}_{3} \oplus \tilde{\mathcal{W}}_{4} \oplus \tilde{\mathcal{W}}_{5} \oplus \tilde{\mathcal{W}}_{7} \oplus \tilde{\mathcal{W}}_{8} \oplus \tilde{\mathcal{W}}_{9} \oplus \tilde{\mathcal{W}}_{10}$

(c) If $2 n \geq 8, \mathfrak{R}(V)=\tilde{\mathcal{W}}_{1} \oplus \tilde{\mathcal{W}}_{2} \oplus \tilde{\mathcal{W}}_{3} \oplus \tilde{\mathcal{W}}_{4} \oplus \tilde{\mathcal{W}}_{5} \oplus \tilde{\mathcal{W}}_{6} \oplus \tilde{\mathcal{W}}_{7} \oplus \tilde{\mathcal{W}}_{8} \oplus \tilde{\mathcal{W}}_{9} \oplus \tilde{\mathcal{W}}_{10}$

We have $\tilde{\mathcal{W}}_{1} \approx \tilde{\mathcal{W}}_{4}$ and, if $2 n \geq 6, \tilde{\mathcal{W}}_{2} \approx \tilde{\mathcal{W}}_{5}$. The other $\tilde{\mathcal{U}}(V,\langle\cdot, \cdot\rangle, J)$ modules appear with multiplicity 1.

(2) We have that:
(a) $\tau \oplus \tau^{\star}: \tilde{\mathcal{W}}_{1} \oplus \tilde{\mathcal{W}}_{4} \underset{\sim}{\stackrel{\approx}{\longrightarrow}} \mathbb{R} \oplus \mathbb{R}$
(b) If $2 n=4, \rho_{0,-, S}: \tilde{\mathcal{W}}_{2} \approx S_{0,-}^{2}\left(V^{*}, \tilde{J}\right)$.
(c) If $2 n \geq 6, \rho_{0,-, S} \oplus \rho_{0,-, S}^{\star}: \tilde{\mathcal{W}}_{2} \oplus \tilde{\mathcal{W}}_{5} \underset{\sim}{\longrightarrow} S_{0,-}^{2}\left(V^{*}, \tilde{J}\right) \oplus S_{0,-}^{2}\left(V^{*}, \tilde{J}\right)$.
(d) $\tilde{\mathcal{W}}_{3}=\{\tilde{A} \in \mathfrak{R}(V): \tilde{A}(x, y, z, w)=-\tilde{A}(\tilde{J} x, \tilde{J} y, z, w) \forall x, y, z, w\}$
$\cap \operatorname{ker}(\rho)$.
(e) If $2 n \geq 8, \tilde{\mathcal{W}}_{6}=\operatorname{ker}\left(\rho \oplus \rho^{\star}\right) \cap\left\{\tilde{A} \in \mathfrak{R}(\tilde{V}): \tilde{J}^{*} \tilde{A}=\tilde{A}\right\} \cap \tilde{\mathcal{W}}_{3}^{\perp}$.
(f) $\tilde{\mathcal{W}}_{7}=\{\tilde{A} \in \mathfrak{R}(V): \tilde{A}(\tilde{J} x, y, z, w)=\tilde{A}(x, y, \tilde{J} z, w) \forall x, y, z, w\}$.
(g) $\rho_{+, S}: \tilde{\mathcal{W}}_{8} \underset{\sim}{\stackrel{\approx}{\longrightarrow}} S_{+}^{2}\left(V^{*}, \tilde{J}\right), \rho_{+, \Lambda}^{\star}: \tilde{\mathcal{W}}_{9} \stackrel{\approx}{\underset{\sim}{\longrightarrow}} \Lambda_{+}^{2}\left(V^{*}, \tilde{J}\right)$.
(h) If $2 n \geq 6, \tilde{\mathcal{W}}_{10}=\left\{\tilde{A} \in \mathfrak{R}(V): \tilde{J}^{*} \tilde{A}=-\tilde{A}\right\} \cap \operatorname{ker}\left(\rho \oplus \rho^{\star}\right)$.

We note for the sake of completeness that $\operatorname{dim}\left(\tilde{\mathcal{W}}_{\nu}\right)=\operatorname{dim}\left(\mathcal{W}_{\nu}\right)$ is given in Equation (5.a).

\section{Almost hyper Hermitian geometry}

6.1. Hyper Hermitian and hyper para Hermitian geometry. Fix a curvature model $\mathfrak{M}=(V,\langle\cdot, \cdot\rangle, A)$. Let $\mathcal{J}:=\left\{J_{1}, J_{2}, J_{3}\right\}$ be a triple of linear maps of $V$. We say that $\mathcal{J}$ is a hyper Hermitian structure if $J_{1}, J_{2}, J_{3}$ are Hermitian structures and if we have the quaternion identities:

$$
J_{1}^{2}=J_{2}^{2}=J_{3}^{2}=- \text { id } \quad \text { and } \quad J_{1} J_{2}=-J_{2} J_{1}=J_{3} .
$$

Similarly, we say that $\mathcal{J}$ is a hyper para Hermitian structure if $J_{1}$ is a Hermitian structure, if $J_{2}$ and $J_{3}$ are para Hermitian structures, and if we have the para quaternion identities:

$$
J_{1}^{2}=-J_{2}^{2}=-J_{3}^{2}=-\mathrm{id} \quad \text { and } \quad J_{1} J_{2}=-J_{2} J_{1}=J_{3} .
$$

Let $\mathfrak{Q}:=(V,\langle\cdot, \cdot\rangle, \mathcal{J}, A)$ be the associated hyper Hermitian curvature model (resp. hyper para Hermitian curvature model). We refer to [43, 44, 48, for further details concerning such structures. We define:

$$
\tau_{\mathfrak{Q}}^{\star}:=\tau_{J_{1}}^{\star}+\tau_{J_{2}}^{\star}+\tau_{J_{3}}^{\star} .
$$


The structure group of a hyper Hermitian structure $\mathcal{J}$ is $S O(3)$ and of a hyper para Hermitian structure is $S O(2,1)$ since we must allow for reparametrizations; $\tau_{\mathfrak{Q}}^{\star}$ is invariant under this structure group and does not depend on the particular parametrization chosen. We say that $(M, g, \mathcal{J})$ is an almost hyper Hermitian manifold or an almost hyper para Hermitian manifold if $\mathcal{J}_{P}$ defines the appropriate structure on $\left(T_{P} M, g_{P}\right)$ for all points $P$ of $M$; we impose no integrability condition.

Theorem 6.1. Let $m \geq 8$.

(1) Any hyper Hermitian curvature model is geometrically realizable by an almost hyper Hermitian manifold of constant scalar and constant $\star$-scalar curvature.

(2) Any hyper para Hermitian curvature model is geometrically realizable by an almost hyper para Hermitian manifold of constant scalar and constant *-scalar curvature.

\section{Hermitian Geometry}

We refer to [2, 4, 45, 59, 75, for additional material on Hermitian geometry. We say an almost Hermitian manifold $\mathcal{M}=(M, g, \mathcal{J})$ is Hermitian if $\mathcal{J}$ is an integrable almost complex structure, i.e. the Nijenhuis tensor

$$
N_{\mathcal{J}}(x, y):=[x, y]+\mathcal{J}[\mathcal{J} x, y]+\mathcal{J}[x, \mathcal{J} y]-[\mathcal{J} x, \mathcal{J} y]
$$

vanishes or, equivalently, in a neighborhood of any point of the manifold there are local coordinates $\left(x_{1}, \ldots, x_{n}, y_{1}, \ldots, y_{n}\right)$ so that

$$
\mathcal{J} \partial_{x_{i}}=\partial_{y_{i}} \text { and } \mathcal{J} \partial_{y_{i}}=-\partial_{x_{i}} .
$$

Similarly [19], we say that $(M, g, \tilde{\mathcal{J}})$ is a para Hermitian manifold if $\tilde{\mathcal{J}}$ is an integrable almost para complex structure, i.e. if the para Nijenhuis tensor $N_{\tilde{\mathcal{J}}}$

$$
N_{\tilde{\mathcal{J}}}(x, y):=[x, y]-\tilde{\mathcal{J}}[\tilde{\mathcal{J}} x, y]-\tilde{\mathcal{J}}[x, \tilde{\mathcal{J}} y]+[\tilde{\mathcal{J}} x, \tilde{\mathcal{J}} y]
$$

vanishes or, equivalently, there exist local coordinates $\left(x_{1}, \ldots, x_{n}, y_{1}, \ldots, y_{n}\right)$ centered at any given point of $M$ so that

$$
\tilde{\mathcal{J}} \partial_{x_{i}}=\partial_{y_{i}} \quad \text { and } \quad \tilde{\mathcal{J}} \partial_{y_{i}}=\partial_{x_{i}}
$$

Gray [4] showed that the curvature tensor of a Hermitian manifold has an additional symmetry given below in Equation (7.a); it is quite striking that a geometric integrability condition imposes an additional algebraic symmetry on the curvature tensor. We refer to [41] for the proof of the first Assertion and to [12] for the proof of the second Assertion in the following Theorem:

\section{Theorem 7.1.}

(1) If a Hermitian curvature model $\mathfrak{C}=(V,\langle\cdot, \cdot\rangle, J, A)$ is geometrically realizable by a Hermitian manifold, then

$$
\begin{aligned}
0 & =A(x, y, z, w)+A(J x, J y, J z, J w) \\
& +A(J x, J y, z, w)+A(x, y, J z, J w)+A(J x, y, J z, w) \\
& +A(x, J y, z, J w)+A(J x, y, z, J w)+A(x, J y, J z, w)\} .
\end{aligned}
$$

(2) If a para Hermitian curvature model $\tilde{\mathfrak{C}}=(V,\langle\cdot, \cdot\rangle, \tilde{J}, \tilde{A})$ is geometrically realizable by a para Hermitian manifold, then

$$
\begin{aligned}
0 & =\tilde{A}(x, y, z, w)+\tilde{A}(\tilde{J} x, \tilde{J} y, \tilde{J} z, \tilde{J} w) \\
& -\tilde{A}(\tilde{J} x, \tilde{J} y, z, w)-\tilde{A}(x, y, \tilde{J} z, \tilde{J} w)-\tilde{A}(\tilde{J} x, y, \tilde{J} z, w) \\
& -\tilde{A}(x, \tilde{J} y, z, \tilde{J} w)-\tilde{A}(\tilde{J} x, y, z, \tilde{J} w)-\tilde{A}(x, \tilde{J} y, \tilde{J} z, w)\} .
\end{aligned}
$$


We refer to 10 for the proof of the first Assertion and to 12 for the proof of the second Assertion in Theorem 7.2, this result provides a useful converse to Theorem 7.1. Again we shall focus on the scalar curvature and the $\star$-scalar curvature:

\section{Theorem 7.2.}

(1) If a Hermitian curvature model $\mathfrak{C}$ satisfies Equation (7.a), then $\mathfrak{C}$ is geometrically realizable by a Hermitian manifold with constant scalar curvature, with constant $\star$-scalar curvature, and with $d \Omega$ vanishing at the realizing point $P$.

(2) If a para Hermitian curvature model $\tilde{\mathfrak{C}}$ satisfies Equation (7.b), then $\tilde{\mathfrak{C}}$ is geometrically realizable by a para Hermitian manifold with constant scalar curvature, with constant $\star$-scalar curvature, and with $d \tilde{\Omega}$ vanishing at the realizing point $P$.

Equation (7.a) is called the Gray identity and Equation (7.b) is called the para Gray identity. The universal symmetries of the curvature tensor of a Hermitian manifold (resp. a para Hermitian manifold) are generated by the Gray (resp. para Gray) identity and the usual curvature symmetries (see Equation (3.a)). This result emphasizes the difference between almost Hermitian and Hermitian manifolds.

Remark 7.3. Since the Hermitian geometric realization can be chosen so that $d \Omega(P)=0$, imposing the Kaehler identity $d \Omega(P)=0$ at a single point imposes no additional curvature restrictions. If $d \Omega=0$ globally, then the manifold is said to be almost Kaehler. This is a very rigid structure, see for example the discussion in [71, and there are additional curvature restrictions. Thus Theorem 7.2 also emphasizes the difference between $d \Omega$ vanishing at a single point and $d \Omega$ vanishing globally.

Remark 7.4. The space of vectors satisfying the Gray condition (resp. the para Gray condition) is exactly $\mathcal{W}_{7}^{\perp}$ (resp. $\left.\tilde{\mathcal{W}}_{7}^{\perp}\right)$ of Theorem 5.2 (resp. Theorem 5.3 ). Furthermore, either the complex Jacobi operator or the complex curvature operator completely determine the components in $\mathcal{W}_{7}^{\perp}$ of a curvature tensor [8]; the algebraic condition determining $\mathcal{W}_{7}$ also plays a role in the study of Jacobi-Ricci commuting curvature tensors 36 .

\section{IVANOV-PETROVA GEOMETRY}

To simplify the discussion, we work in the Riemannian setting; there are analogous results in arbitrary signatures. Let $\mathfrak{M}=(V,\langle\cdot, \cdot\rangle, A)$ be a Riemannian curvature model. Let $\{x, y\}$ be a basis for an oriented 2-plane $\pi$. The skew-symmetric curvature operator $\mathcal{R}(\pi)$ is defined by setting

$$
\mathcal{R}(\pi):=\left\{\langle x, x\rangle\langle y, y\rangle-\langle x, y\rangle^{2}\right\}^{-1 / 2} \mathcal{R}(x, y) .
$$

This skew-symmetric operator is independent of the particular basis chosen. We say that $\mathfrak{M}$ isIvanov-Petrova if the eigenvalues of $\mathcal{R}(\pi)$ are constant on the Grassmannian of oriented 2-planes in $V$. Similarly we say that a Riemannian manifold $\mathcal{M}=(M, g)$ is Ivanov-Petrova if the curvature model $\left(T_{P} M, g_{P}, R_{P}\right)$ is IvanovPetrova for all points $P$ of the manifold.

The study of such manifolds was initiated by Ivanov and Petrova [42] in dimension 4 and the notation "Ivanov-Petrova" was adopted by later authors following this seminal paper. Let $\phi$ be a self-adjoint map, i.e. $\langle\phi x, y\rangle=\langle x, \phi y\rangle$ for all $x, y$. We form the following algebraic curvature tensor:

$$
A_{\phi}(x, y, z, w)=\{\langle\phi x, z\rangle\langle\phi y, z w\rangle-\langle\phi x, w\rangle\langle\phi y, z\rangle\} .
$$


8.1. Ivanov-Petrova curvature models. We have the following examples 33 , 34, 42, in the algebraic context:

\section{Example 8.1.}

(1) Let $\phi$ be a self-adjoint map of $(V,\langle\cdot, \cdot\rangle)$ with $\phi^{2}=$ id. Adopt the notation of Equation (8.a). Then $\left(V,\langle\cdot, \cdot\rangle, C A_{\phi}\right)$ is Ivanov-Petrova for any constant $C$. Note that if $\phi= \pm \mathrm{id}$, then $A_{\phi}$ has constant sectional curvature $C$.

(2) Let $\left\{e_{1}, e_{2}, e_{3}, e_{4}\right\}$ be the standard normalized orthonormal basis for $\mathbb{R}^{4}$. Let $2 a_{1}+a_{2}=0$. Let the non-zero components of $A$, up to the usual symmetries, be:

$$
\begin{array}{llll}
R_{1212}=a_{1}, & R_{1234}=a_{2}, & R_{1313}=a_{2}, & R_{1324}=-a_{1} \\
R_{1414}=a_{2}, & R_{1423}=a_{1}, & R_{2323}=a_{2}, & R_{2314}=a_{1} \\
R_{2424}=a_{2}, & R_{2413}=-a_{1}, & R_{3434}=a_{1}, & R_{3412}=a_{2}
\end{array} .
$$

This tensor is Ivanov-Petrova; we also refer to 33] where this tensor is described in terms of quaternions.

One has a complete classification. The 4 dimensional case is exceptional and is covered by Ivanov and Petrova [42. The cases $m \geq 5$ and $m \neq 7,8$ are dealt with by the work of [34]. The case $m=8$ is treated in [33] and the case $m=7$ is presented in Nikolayevsky [53].

Theorem 8.2. Any Ivanov-Petrova curvature model of dimension $m \geq 4$ is isomorphic to one of the models of Example 8.1.

8.2. Ivanov-Petrova manifolds. When we pass to the geometric setting, we have the following examples [34, 42, of Ivanov-Petrova manifolds:

\section{Example 8.3.}

(1) Any manifold of constant sectional curvature is Ivanov-Petrova.

(2) Let $(N, h)$ be a metric of constant sectional curvature $K \neq 0$. Consider $f(t)=K t^{2}+A t+B$ and let $\mathcal{O}$ be an open subset of $\mathbb{R}$ where $f>0$. Let $M=\mathcal{O} \times N$ with the metric

$$
g=d t^{2}+f(t) h
$$

If $A^{2}-4 K B \neq 0$, this metric does not have constant sectional curvature and is Ivanov-Petrova.

We then have the following geometric classification Theorem [33, 34, 42, 53]:

Theorem 8.4. Any Ivanov-Petrova manifold of dimension $m \geq 4$ is locally isometric to one of the manifolds of Example 8.3.

8.3. Ivanov-Petrova curvature models which are not geometrically realizable by Ivanov-Petrova manifolds. If $\phi$ is a self-adjoint map of $(V,\langle\cdot, \cdot\rangle)$ with $\phi^{2}=\mathrm{id}$, we can find an orthonormal basis $\left\{e_{i}\right\}$ for $V$ so that $\phi e_{i}= \pm e_{i}$. Let $p$ be the number of +1 eigenvalues and $q$ the number of -1 eigenvalues; the modified inner product $\langle x, y\rangle_{\phi}:=\langle\phi x, y\rangle$ has signature $(p, q)$. The curvature tensors of the manifolds of Example 8.3 (1) correspond to $\phi$ with $\langle\cdot, \cdot\rangle_{\phi}$ having signature $(0, m)$ or $(m, 0)$ (i.e. $\phi= \pm \mathrm{id}$ ) and the curvature tensors of Example 8.3 (2) correspond to $\phi$ with $\langle\cdot, \cdot\rangle_{\phi}$ having signature $(1, m-1)$ or $(m-1,1)$. Thus we may combine Theorems 8.2 and Theorem 8.4 to see:

Theorem 8.5. Any Ivanov-Petrova curvature model of dimension $m \geq 4$ is geometrically realizable by an Ivanov-Petrova manifold if and only if it has the form given in Example 8.1 (1) where $\langle\cdot, \cdot\rangle_{\phi}$ has signature $(0, m),(m, 0),(1, m-1)$, or $(m-1,1)$.

Note that the exceptional Ivanov-Petrova model of Example 8.1 (2) in dimension 4 is not geometrically realizable by an Ivanov-Petrova manifold. 


\section{Osserman Geometry}

Fix a curvature model $\mathfrak{M}=(V,\langle\cdot, \cdot\rangle, A)$; again, we restrict to the Riemannian setting. Let $S$ be the unit sphere in $(V,\langle\cdot, \cdot\rangle)$. The Jacobi operator

$$
\mathfrak{J}(x): y \rightarrow \mathcal{R}(y, x) x
$$

is a self-adjoint operator which appears in the study of geodesic sprays. We say that a Riemannian curvature model or a Riemannian manifold is Osserman if the eigenvalues of $\mathfrak{J}$ are constant on $S$.

9.1. Osserman curvature models. Let $\psi$ be a skew-adjoint map of $(V,\langle\cdot, \cdot\rangle)$. Motivated by the splitting $\sigma \rho_{a}$ of Theorem 2.1, we form the algebraic curvature tensor:

$$
A_{\psi}(x, y, z, w):=\langle\psi y, z)\langle\psi x, w\rangle-\langle\psi x, z\rangle\langle\psi y, w\rangle-2\langle\psi x, y\rangle\langle\psi z, w\rangle .
$$

The following examples appear first in [32]:

Example 9.1. Let $\left\{\psi_{1}, \ldots, \psi_{\ell}\right\}$ be a family of skew-adjoint endomorphisms defined on $(V,\langle\cdot, \cdot\rangle)$ which satisfy the Clifford commutation relations:

$$
\psi_{i} \psi_{j}+\psi_{j} \psi_{i}=-2 \delta_{i j}
$$

Let $\left\{\lambda_{0}, \lambda_{1}, \ldots, \lambda_{\ell}\right\}$ be real constants where $\lambda_{i} \neq 0$ if $i>0$. Set

$$
A=\lambda_{0} A_{\mathrm{id}}+\lambda_{1} A_{\psi_{1}}+\ldots+\lambda_{\ell} A_{\psi_{\ell}} .
$$

Then $(V,\langle\cdot, \cdot\rangle, A)$ is an Osserman curvature model. If $\ell=0$, then $A=\lambda_{0} A_{\text {id }}$ has constant sectional curvature.

Remark 9.2. The family $\left\{\psi_{i}\right\}$ is said to give a Clifford module structure to $V$ and the maximal such $\ell$ possible is called the Adams number and is denoted by $\nu(m)$. If $m$ is odd, no such structure is possible and $\nu(m)=0$. If $m \equiv 2 \bmod 4$, then only $\ell=1$ is possible. If $m \equiv 4 \bmod 8$, then $\ell=3$ is possible; this case can be realized by a quaternion structure (although there are other structures possible if $m>4$ ). We refer to Adams 1 for further details as this number is closely related to the number of linearly independent vector fields on spheres. If $m=a 2^{s}$ where $a$ is odd, then $\nu(m)=\nu\left(2^{s}\right)$. We have

$$
\nu(1)=0, \quad \nu(2)=1, \quad \nu(4)=3, \quad \nu(8)=7, \quad \nu\left(16 \cdot 2^{k}\right)=8+\nu\left(2^{k}\right) .
$$

Theorem 9.3. Let $\mathfrak{M}$ be an Osserman curvature model of dimension $m \neq 16$. Then $\mathfrak{M}$ is isomorphic to one of the curvature models of Example 9.1.

Theorem 9.3 was proved by Chi 17 if $m \equiv 1 \bmod 2$, if $m \equiv 2 \bmod 4$, and if $m=4$. Subsequently Nikolayevsky [52, 54, 55] established Theorem 9.3 for the remaining values. The result fails in dimension 16; the curvature model of the Cayley plane is not given by a Clifford module structure and the classification is unknown in that dimension.

9.2. Osserman manifolds. Osserman [56] conjectured that any Riemannian manifold whose Jacobi operator had constant eigenvalues on the set of unit tangent vectors was necessarily a local 2-point homogeneous space, i.e. is either flat or is a rank 1 symmetric space. This conjecture became known as the Osserman conjecture by subsequent authors and thecondition that the Jacobi operator has constant eigenvalues is known as the Osserman condition. The Osserman conjecture in the Riemannian setting has been settled except in dimension 16 where it remains open 17, 52, 54, 55,

Theorem 9.4. If $\mathcal{M}$ is a Riemannian Osserman manifold of dimension $m \neq 16$, then either $\mathcal{M}$ is flat or $\mathcal{M}$ is locally isometric to a rank 1 symmetric space. 
Remark 9.5. Theorem 9.4 fails in the indefinite setting. There are Walker manifolds of signature $(2,2)$ which are Osserman but not locally homogeneous [9, 31].

9.3. Osserman curvature models which are not geometrically realizable by Osserman manifolds. We adopt the notation of Example 9.1. If $\mathfrak{M}$ is the curvature model of a rank 1 symmetric space ot dimension $m \neq 16$, then one of the following cases holds:

(1) $\ell=0$ and $\mathcal{M}$ has constant sectional curvature so $\mathcal{M}$ is locally isometric to a rescaled sphere or hyperbolic space.

(2) $\ell=1$ and $\lambda_{1}=\lambda_{0}$ so $\mathcal{M}$ is locally isometric to a rescaled complex projective space or the negative curvature dual.

(3) $\ell=3$ and $\lambda_{0}=\lambda_{1}=\lambda_{2}=\lambda_{3}$ so $\mathcal{M}$ is locally isometric to a rescaled quaternionic projective space or the negative curvature dual.

The following result now follows from the discussion above:

Theorem 9.6. Let $\mathfrak{M}$ be an Osserman curvature model of dimension $m \neq 16$. Adopt the notation of Example 9.1. Assume $\ell \geq 1$. If $\ell \neq 1,3$ or if $\lambda_{i} \neq \lambda_{j}$ for some $i \neq j$, then $\mathfrak{M}$ is not geometrically realizable by an Osserman manifold.

\section{Curvature homogeneity}

Let $\mathfrak{M}=(V,\langle\cdot, \cdot\rangle)$ be a curvature model. We say that a pseudo Riemannian manifold $\mathcal{M}=(M, g)$ is curvature homogeneous with model $\mathfrak{M}$ if every point of $M$ realizes $\mathfrak{M}$ geometrically; in this situation, $\mathcal{M}$ is said to be curvature homogeneous. Equivalently, this means that given any 2 points $P$ and $Q$ of $M$, there is an isometry $\Phi_{P, Q}: T_{P} M \rightarrow T_{Q} M$ so $\Phi_{P, Q}^{*} R_{Q}=R_{P}$. More generally, if $\Phi_{P, Q}^{*} \nabla^{i} R_{Q}=\nabla^{i} R_{P}$ for $i \leq k$, then $\mathcal{M}$ is said to be $k$ curvature homogeneous. One has the following result of Singer 68] in the Riemannian setting and of Podesta and Spiro [58] in the pseudo Riemannian setting:

Theorem 10.1. There exists an integer $k_{p, q}$ so that if $\mathcal{M}$ is any complete simply connected pseudo Riemannian manifold of signature $(p, q)$ which is $k_{p, q}$ curvature homogeneous, then $\mathcal{M}$ is homogeneous.

One has rigidity results of Tricerri and Vanhecke 73 and of Cahen, Leroy, Parker, Tricerri, and Vanhecke [16]:

\section{Theorem 10.2.}

(1) A Riemannian curvature homogeneous manifold which is 0 curvature modeled on an irreducible symmetric space is locally symmetric.

(2) A Lorentzian curvature homogeneous manifold which is 0 curvature modeled on an irreducible symmetric space has constant sectional curvature.

In the Riemannian setting, [26, 67] there are curvature homogeneous manifolds which are not locally homogeneous but there are no known examples which are 1 curvature homogeneous but not locally homogeneous. Work of 63, 65, shows that any 1 curvature homogeneous complete simply connected Riemannian manifold of dimension $m \leq 5$ is homogeneous. In the Lorentzian setting ( $p=1)$, there exist 1 curvature homogeneous Lorentzian manifolds which are not locally homogeneous 13, 14. On the other hand, given any $k$, one can construct neutral signature pseudo Riemannian manifolds which are complete, which are modeled on a symmetric space, which are $k$ curvature homogeneous, and which are not locally homogeneous 35.

The results of Sections 8 and 9 immediately yield:

Theorem 10.3. There exist Riemannian curvature models which are not geometrically realizable by curvature homogeneous manifolds. 


\section{ACKNOWLEDGMENTS}

Research of M. Brozos-Vázquez was partially supported by Project MTM200601432 (Spain). Research of P. Gilkey partially supported Projects MTM2006-01432 (Spain) and DGI SEJ2007-67810a (Spain). Research of S. Nikčević partially supported by Research of Project 144032 (Serbia).

\section{REFERENCES}

[1] J. Adams, Vector fields on spheres, Annals of Math. 75 (1962), 603-632.

[2] V. Apostolov, G. Ganchev, and S. Ivanov, Compact Hermitian surfaces of constant antiholomorphic sectional curvatures, Proc. Amer. Math. Soc. 125 (1997), 3705-3714.

[3] T. Aubin, Équations différentielles non linéaires et probléme de Yamabe concernant la courbure scalaire, J. Math. Pures Appl. 55 (1976), 269-296.

[4] D. E. Blair, Nonexistence of 4-dimensional almost Kaehler manifolds of constant curvature, Proc. Amer. Math. Soc. 110 (1990), 1033-1039.

[5] N. Blažić, P. Gilkey, S. Nikčević, and U. Simon, Algebraic theory of affine curvature tensors, Archivum Mathematicum (BRNO), 42 (2006), Suppl., 147-168.

[6] N. Bokan, On the complete decomposition of curvature tensors of Riemannian manifolds with symmetric connection, Rend. Circ. Mat. Palermo XXIX (1990), 331-380.

[7] N. Bokan, M. Djorić, and U. Simon, Geometric structures as determined by the volume of generalized geodesic balls, Result. Math. 43 (2003), 205-234.

[8] M. Brozos-Vázquez, E. García-Río, and P. Gilkey, Relating the curvature tensor and the complex Jacobi operator of an almost Hermitian manifold, Adv. Geom. 8 (2008), 353-365.

[9] M. Brozos-Vázquez, E. García-Río, P. Gilkey, S. Nikčević, and R. Vázquez-Lorenzo The Geometry of Walker Manifolds, Morgan and Claypool (to appear).

[10] M. Brozos-Vázquez, P. Gilkey, H. Kang, and S. Nikčević, Geometric realizations of Hermitian curvature models, arXiv:0812.2743

[11] M. Brozos-Vázquez, P. Gilkey, H. Kang, S. Nikčević, and G. Weingart, Geometric realizations of curvature models by manifolds with constant scalar curvature, to appear Differential Geom. Appl., arXiv:0811.1651.

[12] M. Brozos-Vázquez, P. Gilkey, S. Nikčević, and R. Vázquez-Lorenzo, Geometric Realizations of para Hermitian curvature models, arXiv:0902.1697

[13] P. Bueken, and M. Djoric, Three-dimensional Lorentz metrics and curvature homogeneity of order one, Ann. Global Anal. Geom. 18 (2000), 85-103.

[14] P. Bueken, and L. Vanhecke, Examples of curvature homogeneous Lorentz metrics, Classical Quantum Gravity 14 (1997), L93-L96.

[15] J. B. Butruille, Espace de twisteurs d une variété presque hermitienne de dimension 6, Ann. Inst. Fourier (Grenoble) $\mathbf{5 7}$ (2007), 1451-1485.

[16] M. Cahen, J. Leroy, M. Parker, F. Tricerri, and L. Vanhecke, Lorentz manifolds modeled on a Lorentz symmetric space, J. Geom. Phys. 7 (1990), 571-581.

[17] Q. S. Chi, A curvature characterization of certain locally rank one symmetric spaces, J. Differential Geom. 28 (1988), 187-202.

[18] A. Cortés-Ayaso, J. C. Díaz-Ramos, and E. García-Río, Four-dimensional manifolds with non-degenerated self-dual Weyl curvature tensor, Ann. Global Anal. Geom. 34 (2008), $185-193$.

[19] V. Cortés, C. Mayer, T. Mohaupt, and F. Saueressig, Special geometry of Euclidean supersymmetry I: vector multiplets, arXiv:hep-th/0312001.

[20] V. Cruceanu, P. Fortuny, and P. M. Gadea, A survey on paracomplex geometry, Rocky Mount. J. Math. 26 (1996), 83-115.

[21] H. del Rio, and S. Simanca, The Yamabe problem for almost Hermitian manifolds, J. Geom. Anal. 13 (2003), 185-203.

[22] J. Deprez, K. Sekigawa, and L. Verstraelen, Classifications of Kaehler manifolds satisfying some curvature conditions, Sci. Rep. Niigata Univ. Ser. A 24 (1988), 1-12.

[23] J. C. Díaz-Ramos, E. García-Río, and R. Vázquez-Lorenzo, Osserman metrics on Walker 4-manifolds equipped with a para Hermitian structure, Mat. Contemp. 30 (2006), 91-108.

[24] M. Falcitelli, and A. Farolina Curvature properties of almost Hermitian manifolds, Riv. Mat. Univ. Parma V (1994), 301-320.

[25] M. Falcitelli, A. Farinola, and S. Salamon, Almost-Hermitian geometry, Differential Geom. Appl. 4 (1994), 259-282.

[26] D. Ferus, H. Karcher, and H. Münzner, Cliffordalgebren und neue isoparametrische Hyperflächen, Math. Z. 177 (1981), 479-502. 
[27] A. Fino, Almost Kaehler 4-dimensional Lie groups with J-invariant Ricci tensor, Differential Geom. Appl. 23 (2005), 26-27.

[28] P. M. Gadea, and J. A. Oubiña, Homogeneous pseudo-Riemannian structures and homogeneous almost para Hermitian structures, Houston J. Math. 18 (1992), 449-465.

[29] G. Ganchev, and V. Mihova, Kaehler manifolds of quasi-constant holomorphic sectional curvatures, Cent. Eur. J. Math. 6 (2008), 43-75.

[30] G. Ganchev, and V. Mihova, Warped product Kaehler manifolds and Bochner-Kaehler metrics, J. Geom. Phys. 58 (2008), 803-824.

[31] E. García-Río, D. Kupeli, and R. Vázquez-Lorenzo, Osserman manifolds in semiRiemannian geometry, Lect. Notes Math. 1777, Springer-Verlag, Berlin, 2002.

[32] P. Gilkey, Manifolds whose curvature operator has constant eigenvalues at the basepoint, J. Geom. Anal. 4 (1994), 155-158.

[33] P. Gilkey, Riemannian manifolds whose skew-symmetric curvature operator has constant eigenvalues II, Differential Geometry and applications (eds Kolar, Kowalski, Krupka, and Slovak) Publ Masaryk University Brno Czech Republic ISBN 80-210-2097-0 (1999), 73-87.

[34] P. Gilkey, J. V. Leahy, and H. Sadofsky, Riemannian manifolds whose skew-symmetric curvature operator as constant eigenvalues, Indiana Univ. Math. J. 48 (1999), 615-634.

[35] P. Gilkey, and S. Nikčević, Complete $k$-curvature homogeneous pseudo-Riemannian manifolds, Ann. Global Anal. Geom. 27 (2005), 87-100.

[36] P. Gilkey, and S. Nikčević, Pseudo-Riemannian Jacobi-Videv Manifolds, Int. J. Geom. Methods Mod. Phys. 4 (2007), 727-738.

[37] P. Gilkey, and S. Nikčević, Geometrical representations of equiaffine curvature operators, Result. Math. 52 (2008), 281-287.

[38] P. Gilkey, S. Nikčević, and U. Simon, Geometric theory of equiaffine curvature tensors, arXiv:0903.5269

[39] P. Gilkey, S. Nikčević, and D. Westerman, Geometric realizations of generalized algebraic curvature operators, J. Math. Phys. 50, 013515 (2009).

[40] P. Gilkey, S. Nikčević, and D. Westerman, Riemannian geometric realizations for Ricci tensors of generalized algebraic curvature operators, to appear in the Conference Proceedings of the VIII International Colloquium on Differential Geometry (World Sci. Publ. Co.).

[41] A. Gray, Curvature identities for Hermitian and almost Hermitian manifolds, Tôhoku Math. J. 28 (1976), 601-612.

[42] S. Ivanov, and I. Petrova, Riemannian manifold in which the skew-symmetric curvature operator has pointwise constant eigenvalues, Geom. Dedicata 70 (1998), 269-282.

[43] S. Ivanov, and S. Zamkovoy, Parahermitian and paraquaternionic manifolds, Differential Geom. Appl. 23 (2005), 205-234.

[44] H. Kamada, Neutral hyperkähler structures on primary Kodaira surfaces, Tsukuba J. Math. 23 (1999), 321-332.

[45] J. Kim, On Einstein Hermitian manifolds, Monatsh. Math. 152 (2007), 251-254.

[46] F. Manhart, Surfaces with affine rotational symmetry and flat affine metric in $\mathbb{R}^{3}$, Studia Sci. Math. Hungar. 40 (2003), 397-406.

[47] F. Martín Cabrera, Special almost Hermitian geometry, J. Geom. Phys. 55 (2005), 450470 .

[48] F. Martín Cabrera, and A. Swann, Almost Hermitian structures and quaternionic geometries, Differential Geom. Appl. 21 (2004), 199-214.

[49] F. Martín Cabrera, and A. Swann, Curvature of special almost Hermitian manifolds, Pac. J. Math. 228 (2006), 165-184.

[50] A. Mizuhara, and H. Shima, Invariant projectively flat connections and its applications, Lobachevskii J. Math. 4 (1999), 99-107.

[51] A. Moroianu, and L. Ornea, Conformally Einstein products and nearly Kaehler manifolds, Ann. Global Anal. Geom. 33 (2008), 11-18.

[52] Y. Nikolayevsky, Osserman manifolds and Clifford structures, Houston J. Math. 29 (2003), 59-75.

[53] Y. Nikolayevsky, Riemannian manifolds whose curvature operator $R(X, Y)$ has constant eigenvalues, Bull. Austral. Math. Soc. 70 (2004), 301-319.

[54] Y. Nikolayevsky, Osserman manifolds of dimension 8, Manuscripta Math. 115 (2004), $31-53$.

[55] Y. Nikolayevsky, Osserman conjecture in dimension $\neq$ 8, 16, Math. Ann. 331 (2005), $505-522$.

[56] R. Osserman, Curvature in the eighties, Amer. Math. Monthly 97, (1990), 731-756.

[57] U. Pinkall, A. Schwenk-Schellschmidt, and U. Simon, Geometric methods for solving Codazzi and Monge-Ampère equations, Math. Ann. 298 (1994), 89-100. 
[58] F. Podesta, and A. Spiro, Introduzione ai Gruppi di Trasformazioni, Volume of the Preprint Series of the Mathematics Department V. Volterra of the University of Ancona, Via delle Brecce Bianche, Ancona, ITALY (1996).

[59] T. Sato, Examples of Hermitian manifolds with pointwise constant anti-holomorphic sectional curvature, J. Geom. 80 (2004), 196-208.

[60] T. Sato, Almost Hermitian structures induced from a Kaehler structure which has constant holomorphic sectional curvature, Proc. Amer. Math. Soc. 131 (2003), 2903-2909.

[61] P. A. Schirokow and A. P. Schirokow, Affine Differentialgeometrie, Teubner Leipzig (1962).

[62] R. Schoen, Conformal deformation of a Riemannian metric to constant scalar curvature, J. Differential Geom. 20 (1984), 479-495.

[63] K. Sekigawa, H. Suga, and L. Vanhecke, Curvature homogeneity for four-dimensional manifolds, J. Korean Math. Soc. 32 (1995), 93-101.

[64] K. Sekigawa, Almost Hermitian manifolds satisfying some curvature conditions, Proceedings of the First International Workshop on Differential Geometry (Taegu, 1996), 1-11, Kyungpook Natl. Univ., Taegu, 1997.

[65] K. Sekigawa, H. Suga, and L. Vanhecke, Four-dimensional curvature homogeneous spaces, Commentat. Math. Univ. Carol. 33 (1992), 261-268.

[66] U. Simon, A. Schwenk-Schellschmidt, and H. Viesel, Introduction to the affine differential geometry of hypersurfaces, Lecture Notes, Science University of Tokyo 1991.

[67] W. Takagi, On curvature homogeneity of Riemannian manifolds, Tôhoku Math. J. 26 (1974), 581-585.

[68] I. M. Singer, Infinitesimally homogeneous spaces, Commun. Pure Appl. Math. 13 (1960), 685-697.

[69] I. M. Singer and J. A. Thorpe, The curvature of 4-dimensional Einstein spaces, 1969 Global Analysis (Papers in Honor of K. Kodaira), Univ. Tokyo Press, Tokyo, 355-365.

[70] R. Strichartz, Linear algebra of curvature tensors and their covariant derivatives, Can. J. Math, XL (1988), 1105-1143.

[71] Z. Tang, Curvature and integrability of an almost Hermitian structure, Internat. J. Math. 17, (2006), 97-105.

[72] F. Tricerri, and L. Vanhecke, Curvature tensors on almost Hermitian manifolds, Trans. Amer. Math. Soc. 267 (1981), 365-397.

[73] F. Tricerri and L. Vanhecke, Variétés riemanniennes dont le tenseur de courbure est celui d'un espace symétrique riemannien irréductible, C. R. Acad. Sci. Paris, Sér. I 302 (1986), 233-235.

[74] N. Trudinger, Remarks concerning the conformal deformation of Riemannian structures on compact manifolds, Ann. Scuola Norm. Sup. Pisa 22 (1968), 265-274.

[75] L. Vezzoni, On the Hermitian curvature of symplectic manifolds, Adv. Geom. 7 (2007), 207-214.

[76] H. Yamabe, On a deformation of Riemannian structures on compact manifolds, Osaka Math. J. 12 (1960), 21-37.

MB: Department of Mathematics, University of A Coruña, Spain

E-mail address: mbrozos@udc.es

PG: Mathematics Department, University of Oregon, Eugene Or 97403 USA.

E-mail address: gilkey@uoregon.edu

SN: Mathematical Institute, Sanu, Knez Mihailova 35, P.P. 367, 11001 Belgrade, SERBIA

E-mail address: stanan@mi.sanu.ac.rs 